\title{
A Technique for Sidelobe Suppression in OFDM Systems
}

\author{
Ivan Cosovic, Sinja Brandes, and Michael Schnell \\ German Aerospace Center (DLR) \\ Institute of Communications and Navigation \\ Oberpfaffenhofen, 82234 Wessling, Germany \\ ivan.cosovic@dlr.de / sinja.brandes@dlr.de / michael.schnell@dlr.de
}

\begin{abstract}
In this paper, a new technique for sidelobe suppression in orthogonal frequency-division multiplexing (OFDM) systems is proposed and investigated. This technique can be applied to both pure OFDM and OFDM based multiple-access systems to improve spectral efficiency. Moreover, it can be utilized within OFDM based overlay systems to avoid interference towards the legacy systems operating in the same frequency band as the overlay system.

The proposed sidelobe suppression technique is based on subcarrier weighting. The real-valued subcarrier weights are determined in such a way that the sidelobes of the transmission signal are minimized using an optimization algorithm which is capable to take several optimization constraints into account. Results show that sidelobes can be easily suppressed by more than $10 \mathrm{~dB}$ with the proposed technique. These results are achieved by allowing only a moderate loss in bit-error rate performance.
\end{abstract}

\section{INTRODUCTION}

The ever-growing need for wireless communications which provide high data rates entails a substantial demand for new spectral resources and more flexible and efficient use of existing resources. Over the last several years innovative spectrum management strategies have been suggested which allow different systems to share the same frequency band [1][3]. Furthermore, similar spectrum sharing policies have been proposed in recent recommendations by the Federal Communications Commission (FCC) [4] in the USA and by the International Telecommunication Union (ITU) [5] in Europe.

The starting premise of the spectrum sharing concepts is that, nowadays, at any given time and place, a significant part of spectrum is unused due to the rigid spectrum policies. A feasible solution to exploit these spectrum gaps is to deploy an orthogonal frequency-division multiplexing (OFDM) system. An important property of OFDM is its flexibility and adjustability to certain spectrum restrictions. With OFDM it is easily possible to realize transmission systems which do not need a continuous transmission band. Certain frequency areas can be left unused by simply turning off the respective subcarriers in these areas. This makes OFDM especially attractive for the implementation in spectrum sharing systems. As illustrated in Fig.1, a so-called overlay OFDM system can be realized if the existing systems applied in the considered frequency band do not use the whole frequency band, but leave some frequency gaps.

One of the most challenging problems of spectrum sharing systems is their successful co-existence in the same frequency

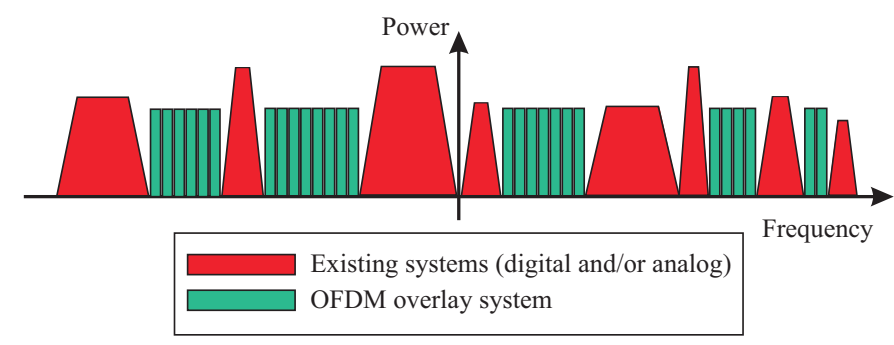

Fig. 1. OFDM overlay concept - using the frequency gaps in an existing frequency bandwidth.

band, i.e., an overlay system should not degrade the performance of the systems already working in that frequency band. Considering OFDM based overlay systems which utilize the frequency gaps of existing systems, out-of-band radiation has to be reduced in order to enable co-existence in the same frequency band. Hence, the suppression of sidelobes is an important task in OFDM based overlay systems.

So far, the topic of sidelobe suppression in OFDM systems has received little attention. An overview of the existing techniques can be found in, e.g., [6]. The first method discussed in [6] is based on the deactivation of subcarriers lying at the borders of the OFDM spectrum, i.e., guard bands are inserted, whereas the second method applies windowing of the transmission signal in frequency domain. However, the first method sacrifices bandwidth and reduces system throughput, whereas the latter method expands the signal in time domain and thus, also, results in a reduced system throughput.

In this paper, a different method to significantly suppress the OFDM sidelobes is proposed and analyzed. This technique, referred to as subcarrier weighting, is based on the multiplication of the used subcarriers with real-valued factors which are chosen such that the sidelobes are suppressed and no side information has to be transmitted. In addition, it overcomes the problems of the techniques presented in [6] as it neither wastes additional scarce spectral resources nor expands the signal in time domain.

The paper is organized as follows. In Section II, the signal model and basic notations are described. In Section III, the subcarrier weighting technique is proposed for sidelobe suppression. To highlight the effectiveness of the presented method, numerical results are given in Section IV. Finally, in Section V conclusions are drawn. 


\section{OFDM SIGNAL MODEL}

To simplify the analysis, an OFDM system having a single continuous transmission band is considered. As illustrated in Fig.1, a real OFDM based overlay system might consist of several transmission sub-bands. Thus, in order to improve sidelobe suppression the proposed algorithm could be applied to the complete OFDM transmission signal instead of to the several continuous transmission sub-bands. However, as we concentrate on the principle of subcarrier weighting in this contribution we leave such investigations for future work.

An OFDM system with a total number of $N$ subcarriers is considered. The block diagram of the OFDM transmitter is illustrated in Fig. 2. The input bits are symbol-mapped applying unit-energy phase-shift keying (PSK) modulation and $N$ complex-valued data symbols $d_{n}$, with $\left|d_{n}\right|^{2}=1, n=$ $1,2, \ldots, N$, are generated. These symbols are serial-to-parallel (S/P) converted resulting in an $N$-element data symbol array $\mathbf{d}=\left(d_{1}, d_{2}, \ldots, d_{N}\right)^{\mathrm{T}}$, where $(.)^{\mathrm{T}}$ denotes transposition. The array $\mathbf{d}$ is fed into the sidelobe suppression unit which outputs $\overline{\mathbf{d}}=\left(\bar{d}_{1}, \bar{d}_{2}, \ldots, \bar{d}_{N}\right)^{\mathrm{T}}$. The sidelobe suppression unit performs the multiplication of each symbol $d_{n}$ with a realvalued weighting factor $g_{n}$. Hence, the entries of $\overline{\mathbf{d}}$ are given by

$$
\bar{d}_{n}=g_{n} d_{n}, n=1,2, \ldots, N .
$$

The weighting factors $g_{n}, n=1,2, \ldots, N$, are chosen such that the sidelobes of the transmission signal are suppressed. Finally, the weighted vector $\overline{\mathbf{d}}$ is modulated on $N$ subcarriers using the inverse discrete Fourier transform (IDFT). After that, parallel-to-serial $(\mathrm{P} / \mathrm{S})$ conversion is performed and a guard interval that exceeds the delay spread of the multipath channel is added as cyclic prefix. Note that throughout this paper it is assumed that the guard interval is much shorter than the useful part of an OFDM symbol.

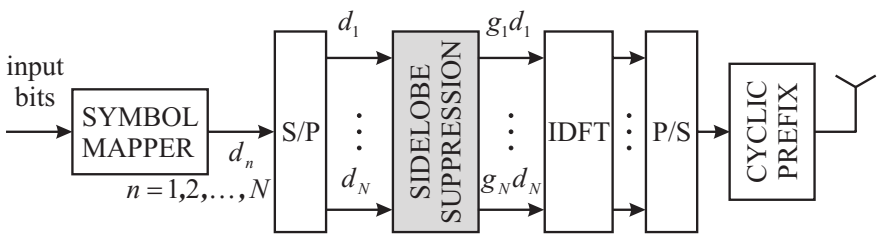

Fig. 2. Block diagram of the OFDM transmitter with sidelobe suppression.

In the following, we aim at the design of the weighting array

$$
\mathbf{g}=\left(g_{1}, g_{2}, \ldots, g_{N}\right)^{\mathrm{T}}, \mathbf{g} \in \mathbf{R}^{N},
$$

according to an optimization criterion by which the sidelobes are suppressed.

\section{Sidelobe SupPression by SubCARrier Weighting}

A single non-weighted subcarrier $s_{n}(x), n=1,2, \ldots, N$, is represented in frequency domain as

$$
s_{n}(x)=d_{n} \frac{\sin \left(\pi\left(x-x_{n}\right)\right)}{\pi\left(x-x_{n}\right)}, n=1,2, \ldots, N .
$$

In (3), $x$ is a normalized frequency given by

$$
x=\left(f-f_{0}\right) T_{0}
$$

where $f$ denotes the frequency, $f_{0}$ is the center frequency, and $T_{0}$ is the OFDM symbol duration excluding the guard interval. In addition, $x_{n}$ is the normalized frequency of the $n$th subcarrier.

As our goal is to suppress the sidelobes in a certain frequency range, we consider $s_{n}(x)$ only in that range. We observe $M$ normalized frequency samples $y_{m}, m=1,2, \ldots, M$, which lie in the frequency range where the optimization of the sidelobes is performed. With that (3) reduces to

$$
\begin{aligned}
& s_{n, m}=s_{n}\left(y_{m}\right)=d_{n} \frac{\sin \left(\pi\left(y_{m}-x_{n}\right)\right)}{\pi\left(y_{m}-x_{n}\right)}, \quad n=1,2, \ldots, N \text {, } \\
& m=1,2, \ldots, M .(5)
\end{aligned}
$$

A simplified frequency domain representation of the nonweighted OFDM signal, the normalized subcarrier frequencies $x_{n}, n=1,2, \ldots, N$, and the normalized frequencies $y_{m}, m=1,2, \ldots, M$, in the optimization range is shown in Fig. 3. For simplicity, a special case is illustrated in which the optimization range is divided in two approximately equal parts which start from the first sidelobes outside the OFDM transmission bandwidth.

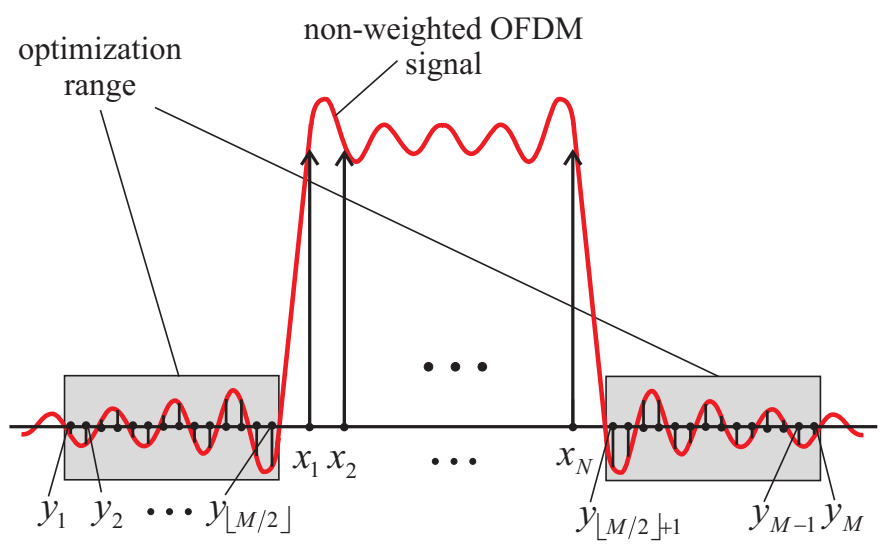

Fig. 3. Simplified frequency domain representation of the non-weighted OFDM signal and the optimization range.

Collecting $s_{n, m}, m=1,2, \ldots, M$, into a vector we obtain $\mathbf{s}_{n}=\left(s_{n, 1}, s_{n, 2}, \ldots, s_{n, M}\right)^{\mathrm{T}}, \quad n=1,2, \ldots, N$. Finally, stacking the vectors $\mathbf{s}_{n}, n=1,2, \ldots, N$, into a matrix we get

$$
\mathbf{S}=\left(\mathbf{s}_{1}, \mathbf{s}_{2}, \ldots, \mathbf{s}_{N}\right) \text {. }
$$

To minimize the sidelobes of the weighted transmission signal $\overline{\mathbf{d}}$, we have to determine the vector $\mathrm{g}$ by solving the following optimization problem

$$
\min _{\mathbf{g}}\|\mathbf{S g}\|^{2}
$$

In addition, we include two constraints on the weighting vector g. The first keeps the transmission power the same as in the case without weighting, i.e.,

$$
\|\overline{\mathbf{d}}\|^{2}=\|\mathbf{d}\|^{2} .
$$


Exploiting (1) and recalling that the transmission symbols $d_{n}$ belong to an unit-energy PSK constellation, i.e., $\left|d_{n}\right|^{2}=1$, (8) reduces to

$$
\|\mathbf{g}\|^{2}=N
$$

The second constraint ensures that the elements of $g$ are between pre-defined limits, i.e.,

$$
g_{\min } \leq g_{n} \leq g_{\max }, n=1,2, \ldots, N .
$$

Such a constraint guarantees that each subcarrier receives a certain amount of the transmission power which is inherently controlled through the ratio $\rho=g_{\max } / g_{\min }$. Furthermore, $g_{\min }$ and $g_{\max }$ can be selected such that a weighted symbol $\bar{d}_{n}$ remains in the same decision region as the original symbol $d_{n}$ as in such case no signalling from transmitter to receiver is required. For example, in the case of PSK this is satisfied for $g_{\min }>0$. Moreover, setting

$$
g_{\min }=1-\frac{\rho-1}{\rho+1} \text { and } g_{\max }=1+\frac{\rho-1}{\rho+1}
$$

ensures that the upper limit, $g_{\max }$, and the lower limit, $g_{\min }$, are equally distant from $\left|d_{n}\right|=1$.

The optimization problem given in (7), together with the constraints in (8) and (10), can be solved numerically. Many effective and reliable numerical methods to solve this optimization problem exist [7].

The principle of the subcarrier weighting technique is illustrated in Fig. 4 for the parameters $N=5, g_{\max } / g_{\min }=$ $\sqrt{4}$, and $\mathbf{d}=(1,1,1,1,1)^{\mathrm{T}}$. The optimization range spans six sidelobes at each side of the used OFDM bandwidth and it starts at the first sidelobe outside the transmission bandwidth. To keep the dimensions of the matrix $\mathbf{S}$ low only one normalized frequency sample per sidelobe is considered in the optimization range, i.e., $M=12$. In addition, these frequencies, $y_{m}, m=1,2, \ldots, M$, are chosen such as to satisfy

$$
\begin{aligned}
\sin \left(\pi\left(y_{m}-x_{n}\right)\right)=1, & n=1,2, \ldots, N, \\
& m=1,2, \ldots, M,
\end{aligned}
$$

i.e., they correspond to the frequencies which lie in the middle between two zero crossings of the individual subcariers. Note that considering more samples in the optimization range can lead to a somewhat better sidelobe suppression, but increases the dimension of the matrix S. For simplicity, in Fig. 4 only the optimization range at the right-hand side of the transmission bandwidth is displayed, whereas the optimization is performed over the complete optimization range. The spectra of the individual subcarriers as well as of the sum signal of all subcarriers are shown in frequency domain. From Fig. 4(a)(b) it can be seen that in the case of subcarrier weighting the signals of the individual subcarriers are adapted so as to mainly cancel each other in the optimization range. As a consequence, in the case of subcarrier weighting the sidelobes of the sum signal exhibit significantly lower values in the optimization range compared to the non-weighted sum signal. Hence, sidelobe suppression is achieved.

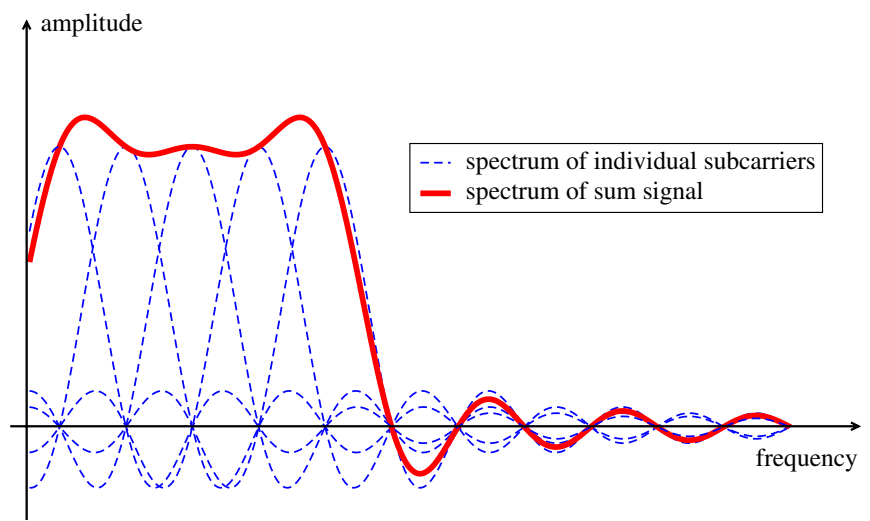

(a)

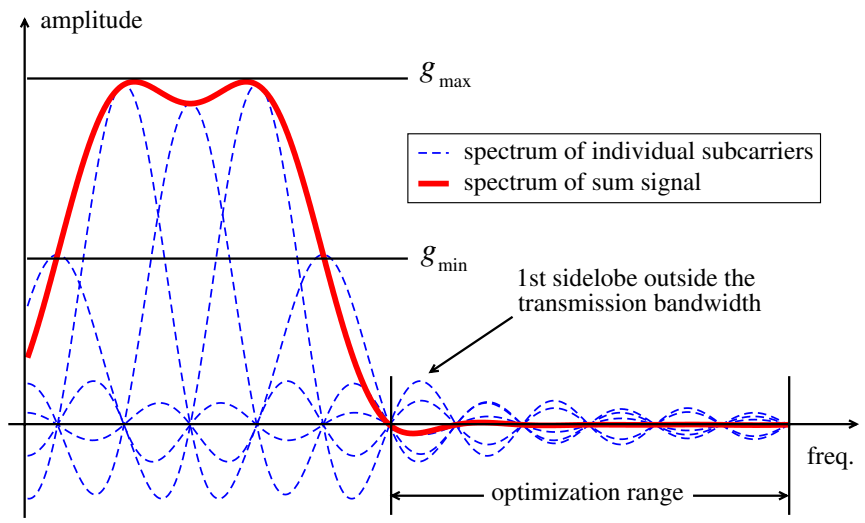

(b)

Fig. 4. Illustration of the subcarrier weighting technique: (a) standard OFDM signal without subcarrier weighting and (b) OFDM signal with subcarrier weighting.

A possible drawback of the subcarrier weighting method is a degradation in bit-error rate (BER) versus signal-to-noise ratio (SNR) performance as, due to the weighting, the subcarriers do not receive equal amounts of transmission power. This degradation depends on the chosen ratio $g_{\max } / g_{\min }$ and is expected to grow as $g_{\max } / g_{\min }$ grows. In the next section the BER performance and the corresponding SNR losses are addressed by numerical simulations assuming different ratios $g_{\max } / g_{\min }$.

\section{NumericAl RESUlts}

In this section, several numerical results are given that illustrate the effectiveness of the subcarrier weighting method for sidelobe suppression.

Binary phase-shift keying (BPSK) modulation is applied and no channel coding is considered. The number of used subcarriers is set to $N=12$. The optimization range consists of 16 sidelobes at each side of the spectrum. Unless otherwise stated, the range starts from the first sidelobe outside the OFDM transmission bandwidth. A single normalized frequency sample is considered per sidelobe in the optimization range, i.e., $M=32$, and the frequency samples $y_{m}, m=1,2, \ldots, M$, are chosen so as to satisfy (12). The ratio $g_{\max } / g_{\min }$ is kept variable throughout the simulations. 
The spectra of the OFDM signals with and without subcarrier weighting are illustrated in Fig. 5 for the symbol vector $\mathbf{d}=(1,1, \ldots, 1)^{\mathrm{T}}$. In the case of subcarrier weighting the ratio of $g_{\max } / g_{\min }=\sqrt{4}$ is used. The benefits of the subcarrier weighting technique are clearly visible. In comparison to OFDM without subcarrier weighting the sidelobes are suppressed by more than $10 \mathrm{~dB}$ in the optimization range.

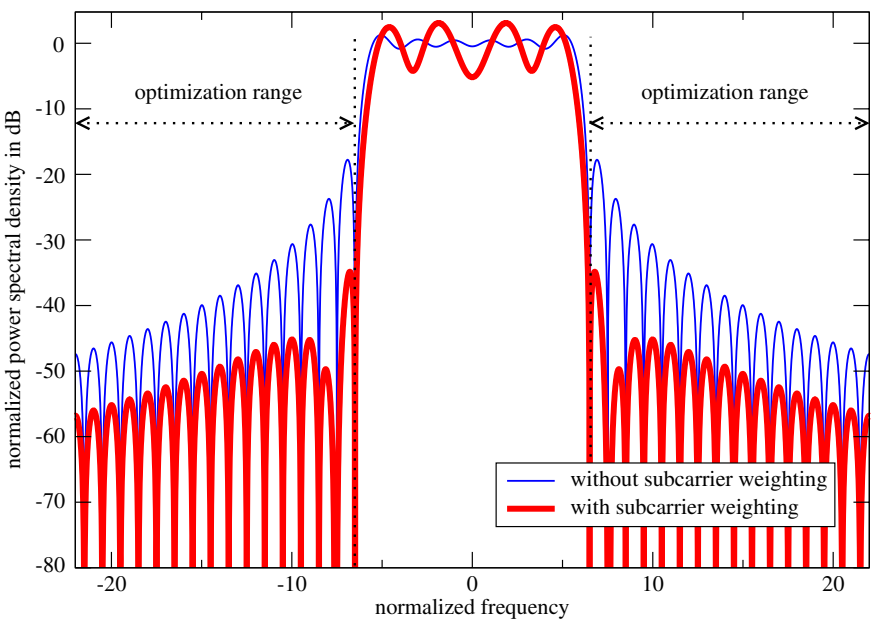

Fig. 5. Spectrum of OFDM signal with and without subcarrier weighting; $N=12 ; M=32 ; \mathbf{d}=(1,1, \ldots, 1)^{\mathrm{T}} ; g_{\max } / g_{\min }=\sqrt{4}$.

The sidelobe suppression averaged over all possible data symbol sequences, i.e., $2^{N}=2^{12}$ sequences, for OFDM applying subcarrier weighting is given in Table I. The simulation results are given for different ratios $g_{\max } / g_{\min }$. To calculate the average sidelobe suppression, standard OFDM without subcarrier weighting is taken as a reference. It is noticeable that already for $g_{\max } / g_{\min }=\sqrt{4}$ a remarkable average suppression of more than $10 \mathrm{~dB}$ is achieved. A further increase of the ratio $g_{\max } / g_{\min }$ enables even better suppression. The reason for this lies in the fact that as this ratio grows the constraint from (10) becomes looser, thus allowing more degrees of freedom to find a solution of (7).

TABLE I

AVERAGE SIDELOBE SUPPRESSION FOR OFDM APPLYING SUBCARRIER WEIGHTING FOR DIFFERENT RATIOS $g_{\max } / g_{\min }$.

\begin{tabular}{|l||c|c|c|c|c|}
\hline ratio $\rho=g_{\max } / g_{\min }$ & 1 & $\sqrt{2}$ & $\sqrt{4}$ & $\sqrt{6}$ & $\sqrt{8}$ \\
\hline $\begin{array}{l}\text { average sidelobe } \\
\text { suppression in } \mathrm{dB}\end{array}$ & 0.0 & 4.9 & 10.21 & 13.45 & 15.76 \\
\hline
\end{tabular}

The OFDM systems applying subcarrier weighting with different ratios $g_{\max } / g_{\min }$ are compared in Fig. 6 on the basis of BER over SNR curves. SNR is given in $E_{\mathrm{b}} / N_{0}$ which represents the energy per transmission bit over the noise spectral density. The underlying mobile radio channel is modelled as a frequency selective Rayleigh fading channel, whereas a maximum ratio combining detector is applied at the receiver assuming perfect channel knowledge. The MonteCarlo simulations are performed taking into account all possible data symbol sequences. As a reference the performance of a standard OFDM system without subcarrier weighting is given. The subcarrier weighting results in an BER loss, since the subcarriers do not receive equal amounts of the transmission power. From (11) it follows that if $g_{\max } / g_{\min }$ grows, $g_{\min }$ becomes lower and thus, some subcarriers receive very small amounts of transmission power and cannot be decoded properly at the receiver resulting in a performance degradation. For example, as shown in Fig. 6, the ratio of $g_{\max } / g_{\min }=\sqrt{6}$ causes an SNR degradation of around 3.2 $\mathrm{dB}$.

The assessment of Table I and Fig. 6 reveals that there are two counteracting effects caused by the ratio $g_{\max } / g_{\min }$. Enlarging this ratio improves sidelobe suppression, but simultaneously leads to a further loss in SNR performance. Therefore, there is a trade-off between the additional sidelobe suppression obtained by enlarging the ratio $g_{\max } / g_{\min }$ and the increased loss in SNR performance. Setting $g_{\max } / g_{\min }=$ $\sqrt{4}$ seems to be a good compromise. A further increase of $g_{\max } / g_{\min }$ is not justified as it leads to a relatively high SNR loss with only moderate improvement in sidelobe suppression.

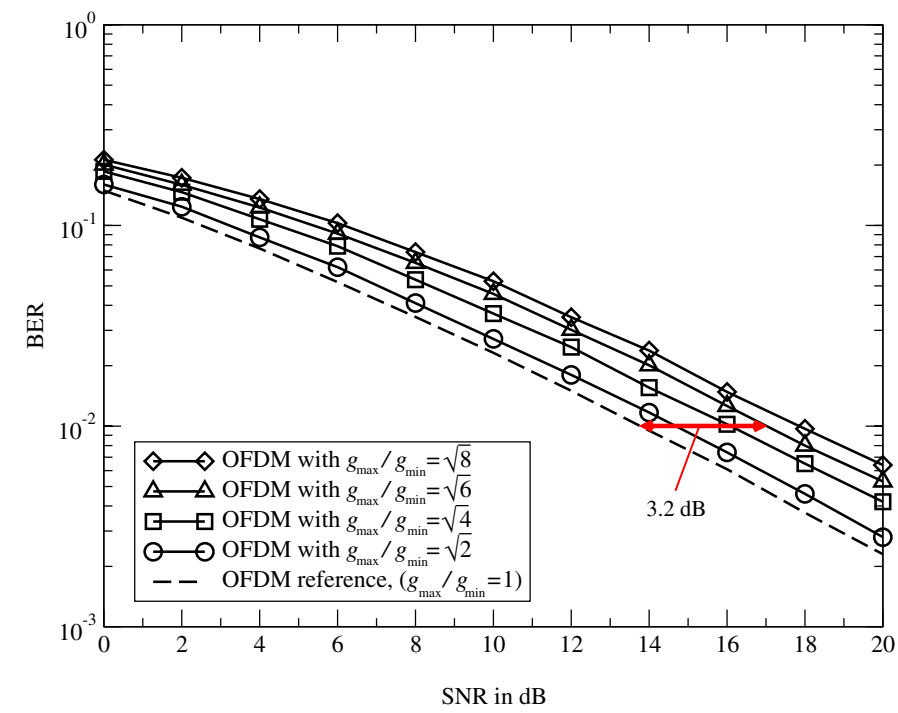

Fig. 6. BER versus SNR for OFDM applying subcarrier weighting; different ratios $g_{\max } / g_{\min } ; N=12$; Rayleigh fading channel; BPSK.

To suppress the sidelobes even further the proposed method can be easily combined with the standard methods described in [6]. As an example we give the results obtained by combining the guard band insertion technique [6] and the subcarrier weighting method.

The spectra of the OFDM signals with and without subcarrier weighting including a guard subcarrier at each side of the transmission bandwidth are illustrated in Fig. 7 for the symbol vector $\mathbf{d}=(1,1, \ldots, 1)^{\mathrm{T}}$. In the case of subcarrier weighting the ratio of $g_{\max } / g_{\min }=\sqrt{4}$ is used. The optimization range starts with the second sidelobe at each side of the spectrum as one subcarrier is left empty at each side of the spectrum, i.e., it is used as a guard subcarrier. As the highest sidelobe, i.e., the first sidelobe, is not attempted to be suppressed with the optimization algorithm, the sidelobes starting with the second 
are suppressed even better than in the case without guard subcarrier (see Fig. 5).

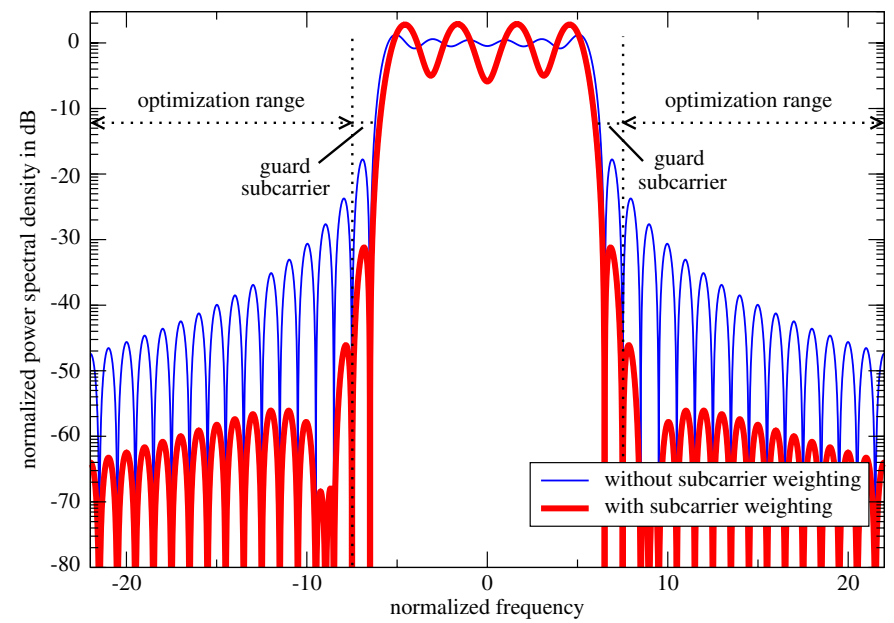

Fig. 7. Spectrum of OFDM signal with and without subcarrier weighting including a guard subcarrier at each side of the transmission bandwidth; $N=$ $12 ; M=32 ; \mathbf{d}=(1,1, \ldots, 1)^{\mathrm{T}} ; g_{\max } / g_{\min }=\sqrt{4}$.

Finally, the sidelobe suppression averaged over all possible data symbol sequences for OFDM applying subcarrier weighting including a guard subcarrier at each side of the transmission band is given in Table II. To obtain these simulation results different ratios $g_{\max } / g_{\min }$ are considered. Again, the optimization range starts with the second sidelobe at each side of the spectrum. As a reference OFDM without subcarrier weighting, but with a guard subcarrier at each side of the transmission band is used. For example, for $g_{\max } / g_{\min }=\sqrt{4}$ the sidelobes are suppressed by more than $13 \mathrm{~dB}$ on the average. In comparison to the corresponding results from Table I, the insertion of a guard subcarrier leads to an additional suppression of about $3 \mathrm{~dB}$.

TABLE II

AVERAGE SIDELOBE SUPPRESSION FOR OFDM APPLYING SUBCARRIER WEIGHTING INCLUDING A GUARD SUBCARRIER FOR DIFFERENT RATIOS $g_{\max } / g_{\min }$.

\begin{tabular}{|l||c|c|c|c|c|}
\hline ratio $\rho=g_{\max } / g_{\min }$ & 1 & $\sqrt{2}$ & $\sqrt{4}$ & $\sqrt{6}$ & $\sqrt{8}$ \\
\hline $\begin{array}{l}\text { average sidelobe } \\
\text { suppression in } \mathrm{dB}\end{array}$ & 0.0 & 6.31 & 13.22 & 17.36 & 20.35 \\
\hline
\end{tabular}

\section{CONCLUSIONS}

In this paper, we have introduced a new technique to suppress the sidelobes of OFDM transmission signals. Besides using this technique to improve the spectral efficiency of OFDM based transmission systems, it can be applied to OFDM based overlay systems to avoid interference towards the legacy systems sharing the same frequency band. The proposed sidelobe suppression scheme does not require transmission of any side information and is capable of reducing the sidelobes of OFDM transmission signals by more than $10 \mathrm{~dB}$. The price to pay for this achievement is a moderate loss in BER performance. Moreover, combining the proposed technique with well-known suppression methods, like e.g. guard band insertion, the suppression capabilities are further increased by several dB. Currently, the proposed sidelobe transmission technique is extended to applications within OFDM based overlay systems.

\section{ACKNOWLEDGMENT}

This work was supported by the Broadband VHF Aeronautical Communications System Based on MC-CDMA (B-VHF) project [8] which is funded by the European Commission within the 6th Framework Programme.

\section{REFERENCES}

[1] J. Zander, "Radio resource management in future wireless networks: Requirements and limitations," in IEEE Communications Magazine, Aug. 1997, pp. 30-36.

[2] J. Mitola, "Cognitive radio for flexible mobile multimedia communications," in Proceedings IEEE International Workshop on Mobile Multimedia Communications, Nov. 1999, pp. 3-10.

[3] T. Weiss and F. Jondral, "Spectrum pooling - an innovative strategy for the enhancement of spectrum efficiency," in IEEE Communications Magazine, Radio Communications Supplement, Mar. 2004, pp. S8-S14.

[4] FCC Spectrum Policy Task Force, Rep. ET Docket no. 02-1935. Federal Communications Commision (FCC), Nov. 2002.

[5] ITU-R, Framework and Overall Objectives of the Future Development of IMT-2000 and Systems Beyond IMT-2000. International Telecommunications Union (ITU); Reccomendation M.1645, June 2003.

[6] T. Weiss, J. Hillenbrand, A. Krohn, and F. Jondral, "Mutual interference in OFDM-based spectrum pooling systems," in Proceedings IEEE Vehicular Technology Conference (VTC'04, Spring), May 2004.

[7] R. Fletcher, Practical Methods of Optimization, 2nd Edition. Wiley, 2000.

[8] http://www.b-vhf.org. 